\title{
Micro-Doppler Classification of Ballistic Threats Using Krawtchouk Moments
}

\author{
Adriano Rosario Persico*, Carmine Clemente*, Luca Pallotta ${ }^{\dagger}$, \\ Antonio De Maio ${ }^{\ddagger}$ and John Soraghan* \\ *University of Strathclyde, CESIP, EEE, 204, George Street, G1 1XW, Glasgow, UK \\ E-mail: adriano.persico, carmine.clemente, j.soraghan- @ strath.ac.uk \\ $\dagger$ CNIT, viale G.P. Usberti, n. 181/A - 43124 Parma, c/o udr Università "Federico II", via Claudio 21, I-80125 Napoli, Italy. \\ E-mail: luca.pallotta@unina.it. \\ ‡Università degli Studi di Napoli "Federico II”, Dipartimento di Ingegneria Elettrica e delle Tecnologie dellInformazione, \\ Via Claudio 21, I-80125 Napoli, Italy. \\ E-mail: ademaio@unina.it.
}

\begin{abstract}
The challenge of ballistic missiles classification is getting greater importance in last years. In fact, since the antimissile defence systems have generally a limited number of interceptors, it is important to distinguish between warheads and confusing objects that the missile releases during its flight, in order to maximize the interception success ratio. For this aim, a novel micro-Doppler based classification technique is presented in this paper characterized by the employment of Krawtchouk moments. Since the evaluation of the latter requires a low computational time, the proposed approach is suitable for real time applications. Finally, a comparison with the 2-dimensional Gabor filter based approach is described by testing both the techniques on real radar data.
\end{abstract}

\section{INTRODUCTION}

The capability to recognize ballistic targets is an important challenge which has appealed greater interest in the last decades. In particular, since during the midcourse phase the missiles are detectable more easily by the defence systems, they release both warheads and some other objects with the aim to confuse the interceptors. Thus they are more expensive than the missiles, then they are available in a limited number for the defence systems. Therefore, it is really important to distinguish between warheads and confusing objects in order to minimize the number of shots per hit and to maximize the ammunition capabilities.

For the specific case of antiballistic missile system, the interceptor are usually equipped with an OnBoard Computer (OBC) to perform control, guidance, target data estimation, mission sequencing and various other critical operations during all the flight, from pre-launch to till impact [1]. However, all these operations are made harder due to the high velocities of the moving target and the interceptor which demands higher data update rates from sensors, high frequent commands to control system. For these reasons fast and low computational classification algorithm is required to exploit in the best way the available resource.

In order to recognize ballistic threats, the micro-Doppler effect analysis, introduced in [2], and widely investigated in the last years [3], may be employed to extract reliable information for target recognition since warheads and confusing objects exhibit different micro-motions during their ballistic trajectory. In fact, while the warhead flight is characterized by precession and nutation, the confusing objects just wobble because they do not have any control phase motor. Analysing the acquired radar signal, these different micro-motions lead to different micro-Doppler signatures, as described in [4] by mathematical model.

Several approaches have been proposed in the literature to extract micro-Doppler features from ballistic missile radar echo. In [5] a method of extracting micro-Doppler of narrowband and wideband signal has been proposed. The method involves the autocorrelation in the time domain and the cepstrum in the frequency domain to extract the period of micro-Doppler variation. In [6] an algorithm for extracting micro-Doppler based feature has been presented. Specifically, starting from a radar signal model for spinning missile, the micro-Doppler modulation frequency has been extracted by analysing the 2-D DFT of a time-frequency distribution (TFD). In particular the short time Fourier Transform (STFT) has been implemented through a data-dependent optimal window length. In [7] the smoothed pseudo Wigner-Ville distribution has been used as high-resolution TFT to calculate micro-Doppler frequency based on the individuation of signal's spectral peaks. The micro-Doppler signature has been used in [8] to extract feature based on the pseudo-Zernike moments for target classification. This approach has been also used successfully to recognize ballistic threats in [9], in which other two different feature extraction approaches are analysed: the 2D (2-dimensional) Gabor filters based features [10] and the Average Cadence Velocity Diagram (ACVD) based features. These three techniques are based on the processing of the Cadence Velocity Diagram (CVD) which represents the cadence of each signal frequency component.

In this paper a novel feature extraction approach for ballistic target classification is presented based on Krawtchouk (Kr) moments. The $\mathrm{Kr}$-moments are widely used for image processing in various applications like image reconstruction [11], shape recognition [12] and face recognition [13] for their some peculiar characteristics. In particular, since they are discretely defined, they do not involve numerical approximation as in the case of continuous orthogonal moments. In this way discretization error does not exist and the amount of resource required to store the polynomials is reduced thanks to the recurrence relations and the symmetry properties of Krawtchouk moments [11]. Moreover, the moments derived from Krawtchouk polynomials benefit of scale, rotation and 
translation invariant properties [11]. These characteristics, together with the capability to pre-compute the polynomials, make this image moments reliable for real time target recognition.

The Kr-moments based approach is compared with the Gabor filter approach which outperforms the other techniques proposed in [9]. The remainder of the paper is organized as follows. Section II describes the relevant theory of the $\mathrm{Kr}$ moments. Section III deals with the classification algorithm while in Section IV the effectiveness of the algorithm is demonstrated on real data. Section $\mathrm{V}$ concludes the paper.

\section{KRAWTCHOUK MOMENTS}

The Krawtchouk moments of order $r$ of an image $I(x, y)$, introduced in [11], are computed as the projection of the image on a basis of orthogonal polynomials which are associated with the binomial distribution. These are calculated as the product of the classical Krawtchouk polynomials and a weight factor to overcome the numerical stability problem as follows [11], [12]

$$
\begin{aligned}
\bar{K}_{r}(x, p, N) & =K_{r}(x, p, N) \sqrt{\frac{w(x ; p, N)}{\rho(n ; p, N)}}= \\
& ={ }_{2} F_{1}\left(-n,-x ;-N ; \frac{1}{p}\right) \sqrt{\frac{w(x ; p, N)}{\rho(n ; p, N)}}
\end{aligned}
$$

with

$$
\begin{aligned}
& w(x ; p, N)=\left(\begin{array}{c}
N \\
x
\end{array}\right) p^{x}(1-p)^{N-x} \\
& \rho(x ; p, N)=(-1)^{N}\left(\frac{1-p}{p}\right)^{n} \frac{n !}{(-N)_{n}}
\end{aligned}
$$

where $x$ and $n$ belonging to $(0,1,2, \ldots, N), N \in \mathbb{N}$, with $\mathbb{N}$ the set of natural numbers, $p$ a real number belonging to the set $(0,1)$. Moreover, ${ }_{2} F_{1}$ is the Gauss hypergeometric function, defined as

$$
{ }_{2} F_{1}(a, b ; c ; z)=\sum_{k=0}^{\infty} \frac{(a)_{k}(b)_{k}}{(c)_{k}} \frac{z^{k}}{k !}
$$

while $(a)_{k}$ is the Pochhammer symbol given by

$$
(a)_{k}=a(a+1) \ldots(a+k-1)=\frac{\Gamma(a+k)}{\Gamma(a)} .
$$

Considering a 2D image $I(x, y)$, the $\mathrm{Kr}$-moments of order $(n, m)$ are defined as [12]

$$
\begin{aligned}
K_{n m}=\sum_{x=0}^{N_{x}-1} \sum_{y=0}^{N_{y}-1} \bar{K}_{n}\left(x, p_{1}, N_{x}-1\right) \times \\
\bar{K}_{m}\left(y, p_{2}, N_{y}-1\right) I(x, y)
\end{aligned}
$$

where $N_{x}$ and $N_{y}$ are the image dimensions along both the axes. Since it is possible to write the image as a series of weighted Krawtchouk polynomials weighted by the $\mathrm{Kr}$ moments, $K_{n m}$, such as [11]

$$
\begin{array}{r}
I(x, y)=\sum_{x=0}^{N_{x}-1} \sum_{y=0}^{N_{y}-1} K_{n m} \bar{K}_{n}\left(x, p_{1}, N_{x}-1\right) \times \\
\bar{K}_{m}\left(y, p_{2}, N_{y}-1\right)
\end{array}
$$

the $\mathrm{Kr}$-moments are a synthetic way to represent the image intensity function $I(x, y)$.

\section{FEATURE EXTRACTION Algorithm}

In this section the micro-Doppler based classification algorithm for ballistic threats is described. Figure 1 shows the block diagram of the classification method in [9] with which the proposed method shares the principal steps. The received



Figure 1: Block diagram of the proposed algorithm.

signal $s_{r x}(n), n=0, \ldots, N$, with $N$ the number of available samples, is pre-processed before the extraction of the microDoppler information. In particular, the first block comprises a notch filtering, down-sampling and normalization with the aim to make clearer the micro-Doppler signature. Then, the preprocessed signal $\tilde{s}_{r x}(n)$ is used to evaluate the spectrogram defined as the modulus of the STFT, as follows

$$
\begin{aligned}
& \chi(\nu, k)=\left|\sum_{n=0}^{N-1} \tilde{s}_{r x}(n) w_{h}(n-k) e^{\left(-j 2 \nu \frac{n}{N}\right)}\right|, \\
& k=0, \cdots, K-1
\end{aligned}
$$

where $\nu$ is the normalized frequency and $w_{h}(\cdot)$ is the smoothing window. Through the spectrogram it is possible to evaluate the signal frequency variations on time. It guarantees robustness with respect to the cross-term interference which characterizes the other common time-frequency distributions (such as the Wigner-Ville distribution) and which may lead to significant errors for the classification. The next step consists in the extraction of the CVD by Fourier transforming the spectrogram along each frequency bin [8], as follows

$$
\Delta(\nu, \varepsilon)=\left|\sum_{k=0}^{K-1} \chi(\nu, k) e^{\left(-j 2 \varepsilon \frac{k}{K}\right)}\right|
$$

where $\varepsilon$ is known as the cadence frequency. The CVD allows to determine the cadence of each frequency component and the maximum Doppler shift, which can be used as discriminant. Moreover, the choice of the CVD guarantees the independence with respect to the initial phase of moving objects instead of the spectrogram. The parameters of the spectrogram are chosen in order to CVD fit a square matrix. The CVD is considered as a 2D image given as input to the feature extraction block which depends on the feature extraction technique. At first, the magnitude of the CVD is normalized in order to obtain a matrix whose values belong to the set $[0,1]$ as follows

$$
\bar{\Delta}(\nu, \varepsilon)=\frac{\Delta(\nu, \varepsilon)-\min _{\nu, \varepsilon} \Delta(\nu, \varepsilon)}{\max _{\nu, \varepsilon}\left[\Delta(\nu, \varepsilon)-\min _{\nu, \varepsilon} \Delta(\nu, \varepsilon)\right]} .
$$

Following, the normalized CVD is processed to extract a $Q$ dimensional feature vector $\boldsymbol{F}=\left[F_{0}, F_{1}, \cdots, F_{Q-1}\right]$, which 
hopefully identifies univocally each class. In the proposed method, the $n m$-th feature is given by (5) replacing the general image $I(x, y)$ with $\bar{\Delta}(\nu, \varepsilon)$ as follows

$$
\begin{aligned}
F_{n m}=\sum_{\nu=0}^{N_{\nu}-1} \sum_{\varepsilon=0}^{N_{\varepsilon}-1} \bar{K}_{n}\left(\nu, p_{1}, N_{\nu}-1\right) \times \\
\bar{K}_{m}\left(\varepsilon, p_{2}, N_{\varepsilon}-1\right) \bar{\Delta}(\nu, \varepsilon)
\end{aligned}
$$

Then, the feature vector $\boldsymbol{F}$ contains the moments for each order up to $(n, m)$ obtaining a vector dimension $Q$ equal to $(n+1)(m+1)$. In order to not affect the classification with polarized vector, $\boldsymbol{F}$ is normalized as follows

$$
\tilde{\boldsymbol{F}}=\frac{\boldsymbol{F}-\eta_{\boldsymbol{F}}}{\sigma_{\boldsymbol{F}}}
$$

where $\eta_{\boldsymbol{F}}$ and $\sigma_{\boldsymbol{F}}$ are the statistical mean and standard deviation of the vector $\boldsymbol{F}$, respectively.

$\mathrm{Kr}$-moments are suitable for this application especially due to their scale invariant property. In fact they guarantee robustness with respect to the aspect angle which affects the maximum Doppler shift which characterizes the CVD. Moreover, since the CVD is characterized by vertical lines whose positions depend on the micro-motions, the application of Kr-moments may be efficient for their capability to extract local characteristics from the image [14].

The classification performances of the extracted feature vectors are evaluated using a modified version of the $k$ Nearest Neighbour $(k \mathrm{NN})$ classifier [9]. The choice of the $k \mathrm{NN}$ classifier is justified for its capability to give as output the scores for each class and for its low computational load. However, it is modified by considering the hypersphere made by the training vectors of each class. In this way, a tested vector is classified as unknown in two cases: if any score of the classes does not overcome the threshold, or if the tested vector does not belong to any hypersphere. Considering the covariance matrix $\mathbf{C}_{c}$ of the training vectors which belong to the class $c$, the hypersphere radius of each class depends on $\sigma_{c}=\operatorname{tr}\left(\mathbf{C}_{c}\right)$. In particular, assuming that the feature vectors are distributed uniformly around their mean vector, for all the Monte Carlo runs, then $r_{c}=\sigma_{c} \sqrt{12} / 2$ [9]. However, the selection of the best classifier is outside the scope of this paper.

\section{EXPERIMENTAL RESULTS}

In this section the presented approach is tested on real data realized by using a reproduction of targets of interest. In particular two different shapes for the warhead have been considered, approximated by a simple cone and a cone with triangular fins at the base, and three for the confusing objects released by the missile, approximated by a cylinder, a cone and a sphere. The dimensions of the targets are comparable. The height and the diameter of the conical and cylindrical targets are 1 and $0.75 \mathrm{~m}$, respectively, while the radius for the spherical confusing object is $1 \mathrm{~m}$. The base and the height of the fins of the warheads instead are equal to 0.20 and $0.50 \mathrm{~m}$, as shown in Figure 2a. Figure 3 shows an example of the set up used for the acquisition of real data. In particular, 10 acquisitions of 10 seconds have been made by using a representative radar for each target and for each possible couple of azimuth and elevation angles with values $\left[0^{\circ}, 45^{\circ}, 90^{\circ}\right]$. Moreover, in order to simulate the different movements of the warheads and the confusing objects it has



(a) Warhead.

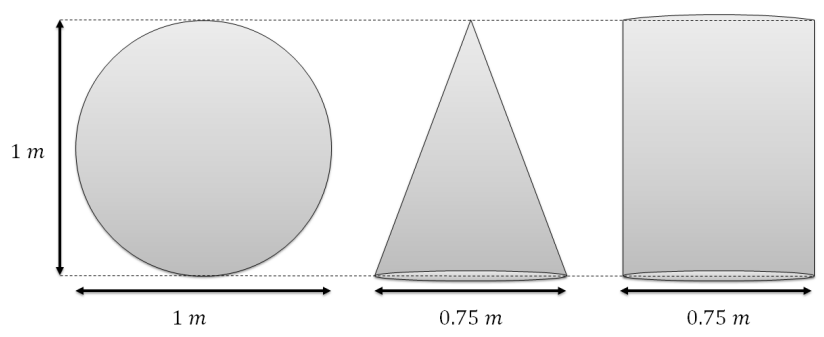

(b) Confusing Object.

Figure 2: Model for the targets.

been used a ST robotic manipulator R-17 [15] (in the blue square in Figure 3) with an added rotor motor.

\section{A. Results}

The targets of interest are divided in two classes: the Warhead class, which comprises two sub-classes, one for warheads without the fins and the other for those with fins, and the Confusing Object class, composed of three sub-classes, each of them for one of the three different considered shapes.

The classification performance are evaluated in terms of three probability: the Probability of correct Classification $\left(P_{C}\right)$, representing the capability to distinguish among the two classes, Warhead and Confusing Objects; the Probability of correct Recognition $\left(P_{R}\right)$, which represents the capability to identify the actual shape of targets within the two principal classes; the Unknown Probability $\left(P_{U}\right)$ given by the ratio of the number of tested vector for which the classifier does not take a decision (as explained in Section III) and the total number of them. In particular a Monte Carlo approach has been used to evaluate the mean values of the three figures of merit over 500 independent runs, in which all the available signals have been divided randomly in $70 \%$ used for training and $30 \%$ for testing.

Both the Kr-moments and the Gabor filter based approach are tested on varying the observation time considering three values which are 10, 5 and 2 seconds, respectively, and on varying the feature vector dimension. Moreover, assuming that the noise is negligible for the acquired signals in a controlled environment, the analysis on the SNR is conducted by adding white Gaussian noise to the real data.

Figures $4 \mathrm{~b}$ and $4 \mathrm{a}$ show the performances of both two methods in terms of $P_{C}$ and $P_{R}$ on varying $Q$, and for a SNR of $0 \mathrm{~dB}$. It is clear that the performance generally improves by increasing the observation time and the number of features. In particular, observing Figure $4 \mathrm{a}$ which shows 

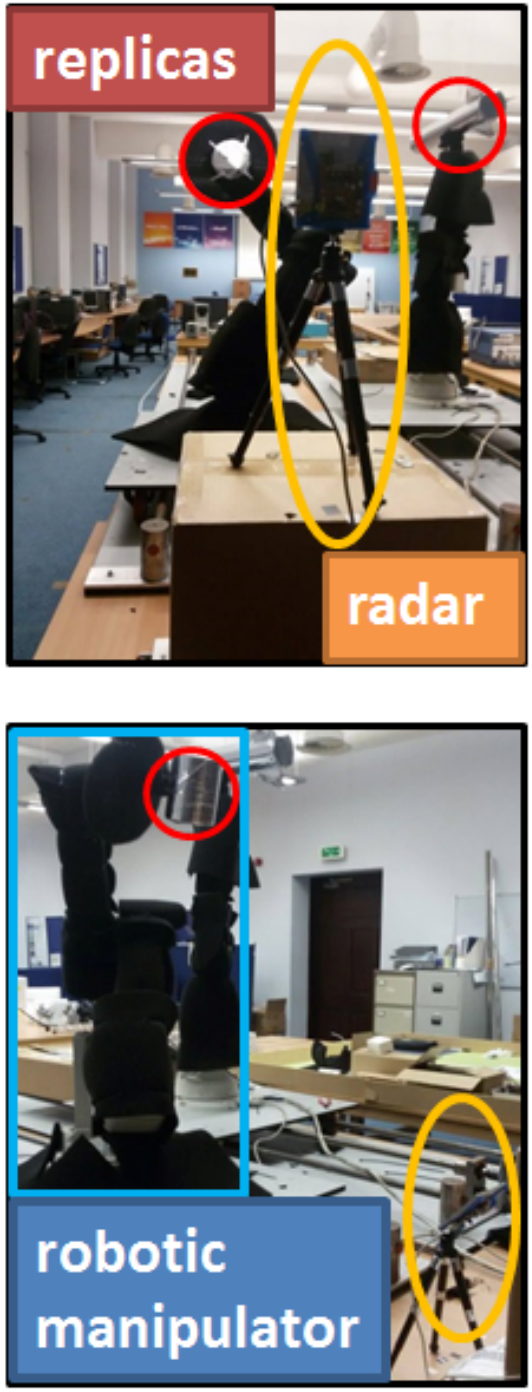

Figure 3: Experiment set up.

the $\mathrm{Kr}$-moments approach performance, it is noted that the gap between $P_{C}$ and $P_{R}$ becomes negligible by increasing $Q$ for signal duration of 10 and 5 seconds. However, $P_{C}$ for signal duration of 2 seconds is still suitable being close to 0.95. From Figure $4 \mathrm{~b}$ instead, it is noted that also for Gabor filter approach considering signal duration of 5 and 10 seconds the gap between the two probability is negligible reaching values greater than 0.98 for all $Q>9$. However, even if the Gabor filter approach generally outperforms the $\mathrm{Kr}$-moments one, the latter guarantees satisfactory values of $P_{C}$ and $P_{R}$, which are greater than 0.95 for highest considered values of $Q$ and observation time.

Figures $5 \mathrm{~b}$ and $5 \mathrm{a}$ show the performances of both the methods in terms of $P_{C}$ and $P_{R}$ on varying the SNR, and for $Q=121$. As the two figures show, the performances for both the approaches are independent of the SNR for signal duration of 5 and 10 seconds and SNR greater than $-5 \mathrm{~dB}$. Figure 6 shows the average running time to extract the feature from the normalized CVD for both the two methods. It is



(a) Kr-moments based approach, $\mathrm{SNR}=0 \mathrm{~dB}$.

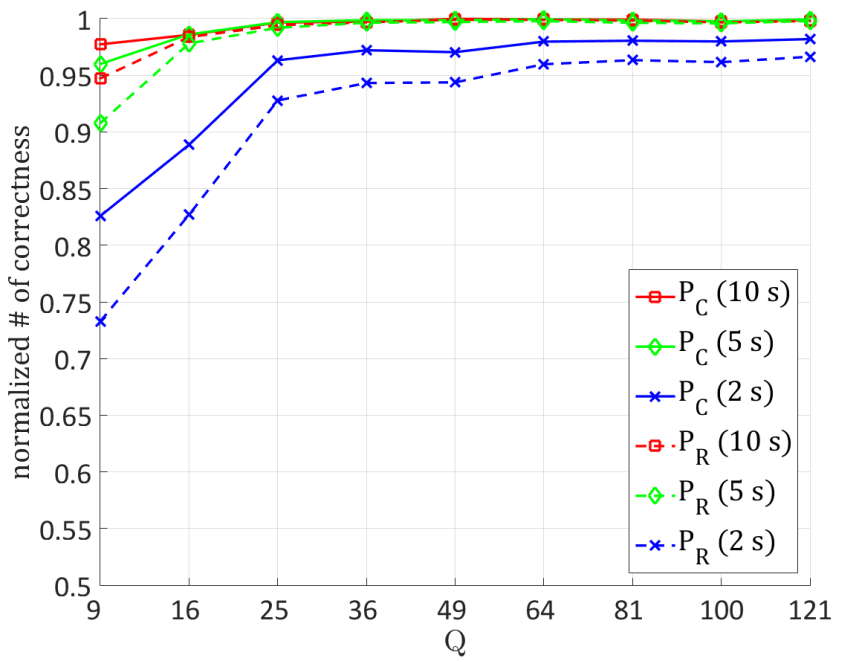

(b) Gabor Filter based approach, SNR $=0 \mathrm{~dB}$.

Figure 4: Probabilities of correct Classification, $P_{C}$, and correct Recognition, $P_{R}$, versus the dimension of the feature vector $Q$ and for several signal's duration; $\mathrm{SNR}=0 \mathrm{~dB}$.

noted that, while for the Gabor filter approach the running time increases as the feature vector dimension increases, for the Kr-moments approach it does not depend on $Q$. Moreover, the running time for $\mathrm{Kr}$-moments is always smaller than for the Gabor filter features of an order of magnitude between 2 and 4 . For this reason, the $\mathrm{Kr}$-moments are more suitable for real time applications or for $\mathrm{OBC}$ of interceptor thanks to their lower amount of required resources and elaboration time combine to satisfactory capability to recognize real threats.

\section{CONCLUSION}

In this paper a novel technique based on the Krawtchouk moments for radar micro-Doppler classification of ballistic threats have been presented. The described approach is compared with the Gabor filter one since they both share the principal steps of the framework. In particular, both the ap- 


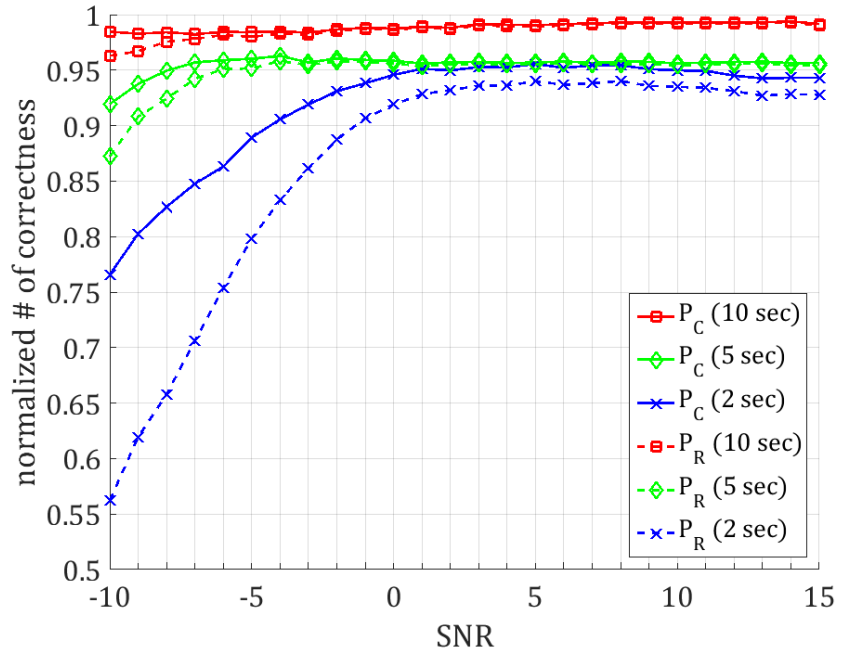

(a) Kr-moments based approach, $Q=121$.



(b) Gabor Filter based approach, $Q=121$.

Figure 5: Probabilities of correct Classification, $P_{C}$, and correct Recognition, $P_{R}$, versus the SNR and for several signal's duration; $Q=121$.

proaches are based on the extraction of reliable information from the normalized CVD to discriminate between warheads and interference factors released by the missile to confuse the defence system. The effectiveness of proposed approach is tested on real micro-Doppler radar data, obtained by acquiring signals scattered by replicas of the targets of interest on varying both the elevation and the azimuth angles using a representative radar. The results have shown that both the two approaches generally allow to discriminate between warheads and confusing objects with a satisfactory degree of correct classification. Moreover, the new approach based on Kr-moments guarantees a very low average running time to evaluate the features which makes it suitable for real time applications such as for the $\mathrm{OBC}$ of interceptor.



Figure 6: Average running time to evaluate Gabor filters features (solid lines) and Kr-moments features (dash lines) versus the feature vector length and for several observation time durations.

\section{ACKNOWLEDGMENT}

This work was supported by the Engineering and Physical Sciences Research Council (EPSRC) Grant number $\mathrm{EP} / \mathrm{K} 014307 / 1$.

\section{REFERENCES}

[1] H. Rathore, M. Katukuri, D. Rao, and B. Narayana Murthy, "Real-time embedded software design for onboard computer of anti-ballistic missile system," in Computer and Communications Technologies (ICCCT), 2014 International Conference on, Dec 2014, pp. 1-5.

[2] V. Chen, F. Li, S. Ho, and H. Wechsler, "Micro-Doppler effect in radar: Phenomenon, model, and simulation study," IEEE Transactions on Aerospace and Electronic Systems, vol. 42, no. 1, pp. 2-21, Jan 2006.

[3] C. Clemente, A. Balleri, K. Woodbridge, and J. Soraghan, "Developments in target micro-Doppler signatures analysis: radar imaging, ultrasound and through-the-wall radar," EURASIP Journal on Advances in Signal Processing, vol. 2013, no. 1, 2013.

[4] H. Gao, L. Xie, S. Wen, and Y. Kuang, "Micro-doppler signature extraction from ballistic target with micro-motions," IEEE Transactions on Aerospace and Electronic Systems, vol. 46, no. 4, pp. 1969-1982, Oct 2010.

[5] Z. Sun, B. Li, and Y. Lu, "Research on micro-motion and micro-doppler of ballistic targets," in Radar Conference, 2009 IET International, April 2009, pp. 1-4.

[6] L. Liu, D. McLernon, M. Ghogho, and W. Hu, "Micro-doppler extraction from ballistic missile radar returns using time-frequency analysis," in Wireless Communication Systems (ISWCS), 2010 7th International Symposium on, Sept 2010, pp. 780-784.

[7] H. x. Sun and Z. Liu, "Micro-doppler feature extraction for ballistic missile warhead," in Information and Automation, 2008. ICIA 2008. International Conference on, June 2008, pp. 1333-1336. 
[8] C. Clemente, L. Pallotta, I. Proudler, A. De Maio, J. Soraghan, and A. Farina, "Pseudo-zernike-based multi-pass automatic target recognition from multi-channel synthetic aperture radar," Radar, Sonar Navigation, IET, vol. 9, no. 4, pp. 457-466, 2015.

[9] A. Persico, C. Clemente, C. Ilioudis, D. Gaglione, J. Cao, L. Pallota, A. De Maio, I. Proudler, and J. Soraghan, "On model and algorithms for micro-doppler based recognition of ballistic targets," IEEE Transactions on Aerospace and Electronic Systems, Under Review.

[10] A. Persico, C. Clemente, C. Ilioudis, D. Gaglione, J. Cao, and J. Soraghan, "Micro-doppler based recognition of ballistic targets using $2 \mathrm{~d}$ gabor filters," in Sensor Signal Processing for Defence (SSPD), 2015, Sept 2015, pp. 1-5.

[11] P.-T. Yap, R. Paramesran, and S.-H. Ong, "Image analysis by krawtchouk moments," Image Processing, IEEE Transactions on, vol. 12, no. 11, pp. 1367-1377, Nov 2003.

[12] B. Kaur, G. Joshi, and R. Vig, "Analysis of shape recognition capability of krawtchouk moments," in Computing, Communication Automation (ICCCA), 2015 International Conference on, May 2015, pp. 1085-1090.

[13] A. Nor'aini and P. Raveendran, "Improving face recognition using combination of global and local features," in Mechatronics and its Applications, 2009. ISMA '09. 6th International Symposium on, March 2009, pp. 1-6.

[14] A. Sit and D. Kihara, "Comparison of image patches using local moment invariants," IEEE Transactions on Image Processing, vol. 23, no. 5, pp. 2369-2379, May 2014.

[15] S. A. P. Ltd., "Robotics within reach," Tech. Rep. [Online]. Available: http://www.strobots.com/images/Brochure.pdf 